\title{
FLUCTUACIÓN POBLACIONAL DE ÁCAROS ASOCIADOS A MANZANO Y PERAL EN EL LLANO CENTRAL DE LA IX REGIÓN DE LA ARAUCANÍA, CHILE
}

\author{
POPULATION FLUCTUATION OF MITES ASSOCIATED WITH APPLE AND PEAR \\ TREES IN THE CENTRAL PLAIN OF THE IX REGION OF LA ARAUCANIA, CHILE
}

\author{
Vivian Medel M.1,2; Ramón Rebolledo R. ${ }^{2}$; Carlos Klein $\mathrm{K}^{4}{ }^{4}$ Alfonso Aguilera $P^{2,3}$
}

\begin{abstract}
RESUMEN
Se estudió la dinámica poblacional de ácaros fitófagos y depredadores en un huerto de manzanos y perales sin aplicación reciente de pesticidas, ubicado en el llano central de la IX Región de La Araucanía con el objetivo de conocer su diversidad y abundancia relativa. Para este propósito se recolectaron, en forma manual, hojas de manzano y peral a dos alturas por árbol (alto y medio). Por cada altura se recolectaron 15 hojas (30 por árbol) con tres repeticiones por especie frutal. La colecta se llevó a cabo durante las temporadas 2000 a 2001 y 2001 a 2002. Los resultados obtenidos muestran que la diversidad fue similar en ambos frutales, encontrándose tres especies de arañitas fitófagas de la familia Tetranychidae Tetranychus urticae (Koch), Panonychus ulmi (Koch) y Bryobia rubrioculus (Scheuten) y solamente un ácaro de la familia Phytoseiidae Chileseus camposi González y Schuster. Además de los ácaros antes mencionados, se encontraron también altas poblaciones de ácaros tideidos y acarididos. La abundancia relativa de los ácaros fitófagos, fitoseidos, acarididos y huevos de ácaros fitófagos fue mayor en manzano que en peral, encontrándose en este último mayor cantidad de ácaros tideidos. No se encontraron diferencias significativas en relación a la abundancia en los diferentes estratos (alto y medio) en ambas especies frutales.

Palabras clave: Tetranychus urticae, Panonychus ulmi, Bryobia rubrioculus, Phytoseiidae Chileseus camposi, diversidad y abundancia relativa.

ABSTRACT

The population dynamics of phytophagous and predatory mites was studied in a pear and apple orchard (without the application of recent pesticides), located in the central plain of La Araucanía (Chile), with the objective to determine mite diversity, as well as their abundance. For this purpose, pear and apple leaves were manually collected at two levels (medium and high). At each level 15 leaves (30 per tree) were collected and sampled with three repetitions per fruit species. The study was carried out during the season of 2000-2001 and 2001-2002. According to the results obtained, the diversity is similar for both fruit species. Three phytophagous Tetranychidae Tetranychus urticae, Panonychus ulmi and Bryobia rubrioculus were found, but only one predator the Chileseus camposi (Phytoseiidae). High populatios of Tydeidae and Acarididae mites were also found. The relative abundance of phytophagous mites and eggs, as well predators and the Acarididae mites were all higher on apples than pears, but on the latter more Tydeidae mites were observed. No significant differences were found regarding the abundance of mites at the two levels studied.

Key words: Tetranychus urticae, Panonychus ulmi, Bryobia rubrioculus, Phytoseiidae, Chileseus camposi, diversity and relative abundance.
\end{abstract}

\section{INTRODUCCIÓN}

Los ácaros fitófagos constituyen importantes plagas en cultivos de manzanos y perales dada su gran polifagia y agresiva estrategia alimenticia que les permite consumir las plantas sobre las cuales se desarrollan y dispersarse eficazmente en busca de nuevos hospederos (González, 1989; García-Marí et al., 1994a; Viñuela y Jacas, 1998; Escudero et al., 1999; Apablaza, 2000).

1 INACAP, Universidad Tecnológica de Chile. Sede Temuco, Chile.

2 Universidad de La Frontera, Facultad de Ciencias Agropecuarias y Forestales. Casilla 54-D. Temuco, Chile.

3 Centro Regional de Investigación INIA Carillanca. Temuco, Chile.

4 Investigador independiente, Casilla 767, Temuco.

Fecha de Recepción: 24 Marzo 2006

Fecha de Aceptación: 28 Abril 2006 
La presencia de "arañitas", como son conocidos vulgarmente los ácaros pertenecientes a la familia Tetranychidae, depende de varios factores, tales como humedad, temperatura y vigor de la planta (García-Marí et al., 1994; Viñuela y Jacas, 1998).

Las especies de tetraníquidos registradas para el país y la zona sur, infestando manzanos y perales, corresponden a Panonychus ulmi (Koch), arañita roja europea; Tetranychus urticae (Koch), arañita bimaculada, y Bryobia rubrioculus (Scheuten), arañita parda de los frutales (González, 1980, 1981a y b, 1984, 1989; Prado, 1991; CostaComelles et al., 1994; Apablaza, 2000; Klein y Waterhouse, 2000). Al respecto, en la IX Región de La Araucanía han estudiado este grupo Salazar et al. $(1998,2000)$, pero enfocado al cultivo de frambueso, y Rebolledo et al. (2004) quienes trabajaron sobre manzano.

Entre los principales depredadores de estos ácaros se encuentran las especies pertenecientes a la familia Phytoseiidae (García-Marí et al., 1990, 1991; Pérez, 1997; Pérez et al., 1999; Viñuela y Jacas, 1998; Iraola et al., 1999). El aumento de las poblaciones de ácaros fitófagos desde hace una treintena de años ha originado un espectacular incremento de los trabajos relacionados con los ácaros depredadores. Se sabe que éstos juegan un importante papel regulador de poblaciones y que los tratamientos fitosanitarios eliminan los potenciales antagonistas, permitiendo de esta manera que los ácaros fitófagos desarrollen una elevada capacidad de multiplicación, así como la adquisición de resistencia frente a los acaricidas (Doreste, 1984; Sánchez, 1994; Cichón et al., 1996).

En la IX Región de La Araucanía los manzanos y perales han adquirido mayor relevancia por el aumento de su superficie, principalmente de productos de exportación, para el consumo fresco y agroindustria, constituyendo además una mayor fuente laboral. Sin embargo, a pesar de las proyecciones de este rubro frutícola, en La Araucanía son escasos los antecedentes sobre la biología de los ácaros tetraníquidos, por ello, el presente trabajo tuvo como objetivos a) determinar las especies de ácaros fitófagos y depredadores y b) determinar la fluctuación poblacional y abundancia relativa de los ácaros fitófagos y ácaros depredadores en manzano y peral.

\section{MATERIALES Y MÉTODOS}

El estudio se llevó a cabo en la ex Estación Experimental Maipo perteneciente a la Universidad de La Frontera, ubicada en el sector urbano de la ciudad de Temuco ( $38^{\circ} 44^{\prime}$ latitud sur y $72^{\circ} 35^{\prime}$ longitud oeste a $100 \mathrm{msnm}$ ).

Los muestreos, con una periodicidad de 15 días, fueron realizados durante las temporadas de noviembre 2000 a mayo 2001 y de diciembre 2001 a mayo 2002 en huertos de manzano y perales de aproximadamente 18 años, los cuales no recibieron tratamiento con plaguicidas los últimos tres años, ni durante el periodo que duró este estudio.

Para el muestreo se eligieron tres árboles más o menos homogéneos, los que a su vez se dividieron en dos alturas (estratos), 1,40 metros (medio) y 1,70 (superior), colectando en cada uno de los estratos 15 hojas en forma aleatoria, para completar un total de 30 hojas por árbol y 180 por cada muestreo.

Las hojas fueron depositadas en bolsas plásticas previamente identificadas y llevadas al Laboratorio de Entomología Aplicada de la Facultad de Ciencias Agropecuarias y Forestales de la Universidad de La Frontera. Una vez en el laboratorio, las muestras se mantenían en un refrigerador entre $2 \mathrm{a} 4^{\circ} \mathrm{C}$ para su posterior revisión. Las hojas eran revisadas por el haz y envés bajo lupa estereoscópica binocular, estableciendo el número de ácaros depredadores (Phytoseiidae) y fitófagos (Tetranychidae) y sus diferentes estados de desarrollo (huevo, larva, ninfa y adulto). Los ácaros muertos y dispersos en las bolsas no fueron contabilizados.

Todo el material fue identificado, en conjunto, por el equipo de trabajo y el material de Phytoseiidae fue, además, enviado a la especialista ingeniero agrónoma señora Lourdes Peralta del SAG Curicó para confirmar la identificación hecha por los autores.

El diseño experimental utilizado fue de bloques completos al azar con tres repeticiones, y los resultados de la fluctuación poblacional se evaluaron mediante un análisis de correlación no paramétrica de Spearman. A su vez, para evaluar la abundancia de las especies de ácaros encontrados en los dos sustratos se aplicó la prueba U de Mann Whitney. La información climática requerida para este estudio fue obtenida en la Estación Meteorológica Aeronáutica de Maquehue, dependiente de la Dirección de Aeronáutica de Chile, ubicada aproximadamente a tres kilómetros en dirección suroeste del lugar de estudio. 


\section{RESULTADOS Y DISCUSIÓN}

En este estudio se encontraron diversas especies de tetraníquidos, tanto en manzano como en peral, existiendo solamente diferencias en la abundancia relativa de los mismos. Las especies correspondieron a Tetranychus urticae (Koch), Panonychus ulmi (Koch) y Bryobia rubrioculus (Scheuten), coincidiendo con Rebolledo et al. (2004), pero difiriendo con Guajardo (1993), quien en un huerto de manzanos de la X Región de Los Lagos encontró solamente a Panonychus ulmi y no las otras dos especies. Al respecto, González (1981) y Sazo (1995) coinciden en que las especies de tetraníquidos más importantes en estos frutales corresponden a T. urticae y P. ulmi.

Además de los ácaros tetraníquidos encontrados, se observaron también ácaros tideidos y acarididos. Con respecto a estos últimos, Ripa y Rodríguez (1999) consideran que la presencia de estos ácaros puede ser beneficiosa al servir como alimento para los ácaros fitoseidos cuando los ácaros fitófagos son escasos.

En ambas temporadas de muestreo se encontró una sola especie de ácaro depredador perteneciente a la familia Phytoseiidae, el cual correspondió a Chileseus camposi González y Schuster colonizando tanto hojas de manzano como de peral, coincidiendo con Guajardo (1993) y Rebolledo et al. (2004). Es necesario destacar que los autores mencionados trabajaron sólo con manzano. En ambos frutales no se registró la presencia de Amblyseius fruticolus González y Schuster, a pesar de que González y Schuster (1962) lo citan desde Valparaíso hasta Temuco. Rebolledo et al. (2004) tampoco lo registraron. Sin embargo, Guajardo (1993) sí lo reportó para la Región de Los Lagos.

La fluctuación poblacional de los ácaros durante la temporada 2000-2001 en peral mostró poblaciones notoriamente bajas de noviembre a abril (Figura 1). Cabe hacer mención que en esta temporada los ácaros fitoseidos y acarididos fueron

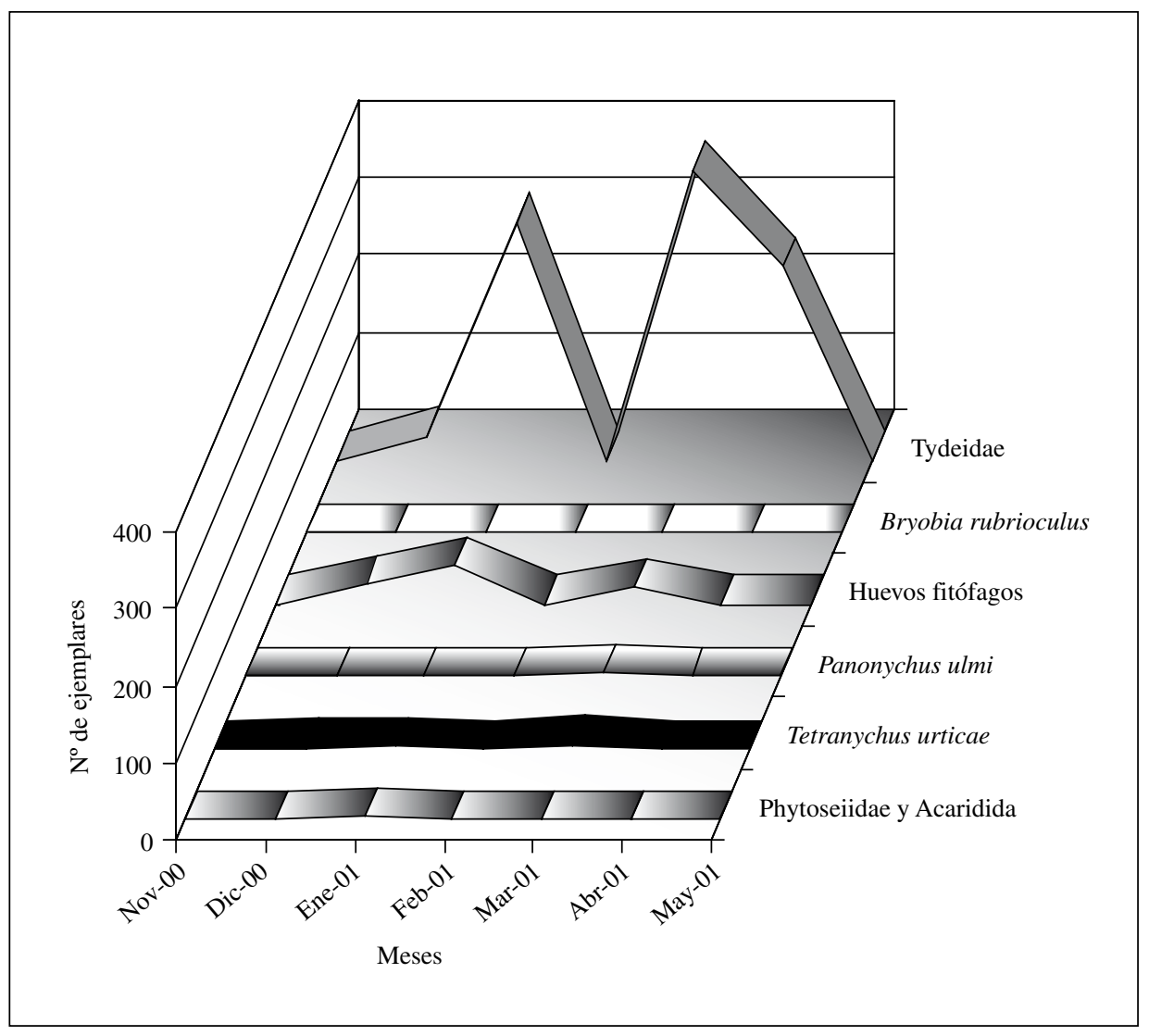

Figura 1. Fluctuación poblacional de ácaros en peral. Temporada 2000-2001. 
contabilizados en conjunto, debido a que estos últimos podrían ser ácaros depredadores. Desde noviembre a enero existió un notable aumento de las poblaciones llegando hasta 365 ejemplares, de los cuales 307 correspondieron a ácaros tideidos, cinco a fitoseidos, dos a Tetranychus urticae y dos a Panonychus ulmi. No se encontraron ejemplares de Bryobia rubrioculus y sólo se contabilizaron 49 huevos de ácaros fitófagos. En febrero, las poblaciones disminuyeron radicalmente, llegando a un ejemplar de fitoseido. Posteriormente, en marzo hubo un importante aumento a 406 individuos, de los cuales 373 correspondieron a tideidos, cinco a T. urticae y un solo un ejemplar de fitoseido. Al final de la colecta sólo se encontraron ejemplares de tideidos, correspondiendo a 250 individuos (Figura 1). Sin duda que la presencia del chape del cerezo (Caliroa cerasi L.) pudo haber tenido un importante efecto, pues el ataque de este himenóptero tentrendínido hizo que los perales prácticamente no tuvieran hojas al final del verano. Esto se contrapone a lo encontrado por Sazo (1995) quien cita para la zona central del país a $P$. ulmi y T. urticae como importantes plagas en peral.

Con respecto a la temporada 2001-2002, en peral (Figura 2) la población de ácaros fue notoriamente mayor, aumentando gradualmente de 47 ejemplares en diciembre hasta 656 ejemplares en el mes de febrero. En marzo hubo una baja considerable, llegando a 151 individuos, de los cuales 121 correspondieron a tideidos, siendo estos ácaros los que se presentaron con mayor abundancia relativa en ambas temporadas. Los huevos de ácaros fitófagos fueron 26 y sólo se encontró un ácaro fitoseido. Durante el mes de abril ocurrió un aumento de la población llegando a 358 individuos, de los cuales sólo uno correspondió a fitoseido. Es importante destacar que a finales del verano en ambas temporadas fue

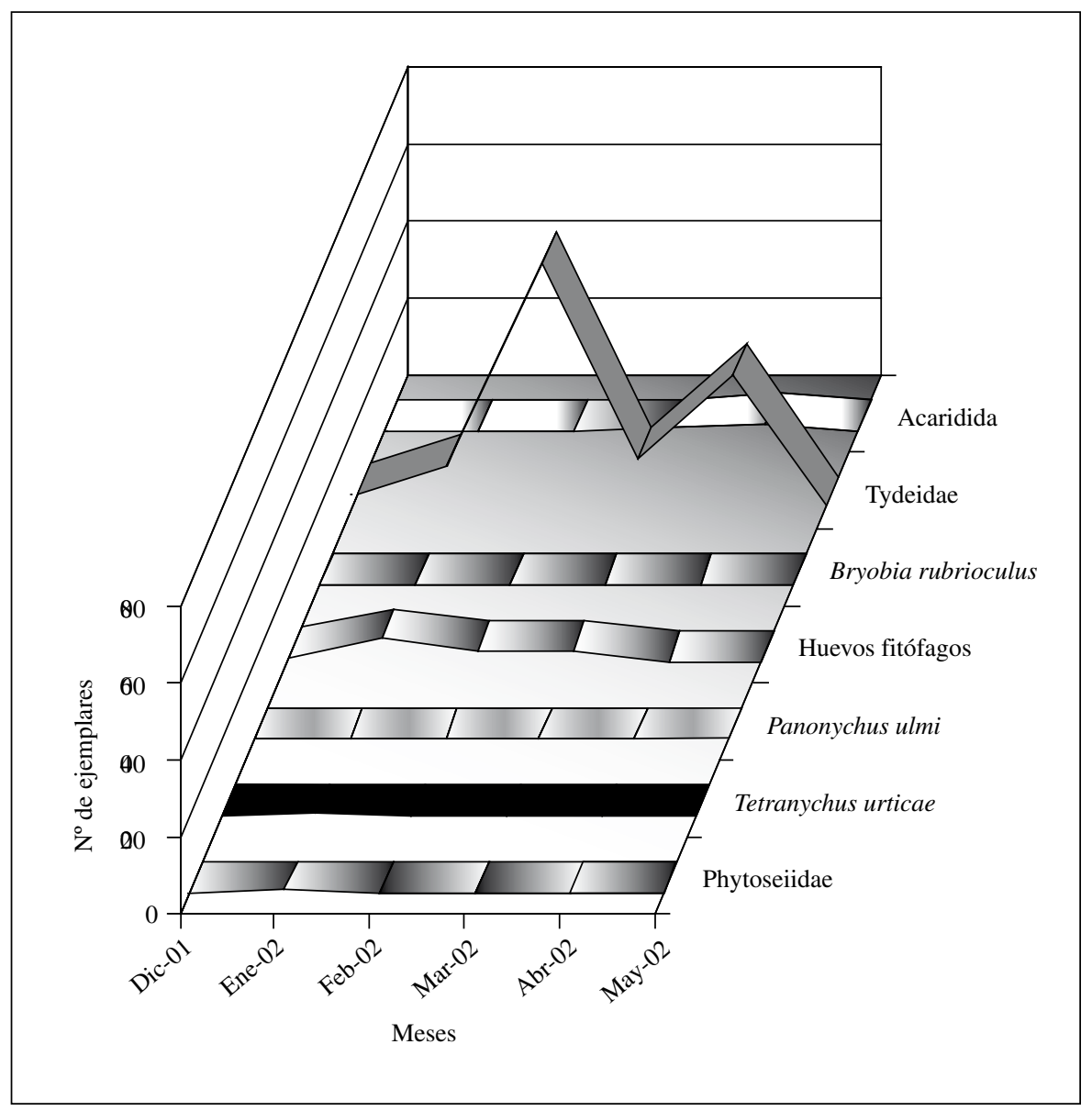

Figura 2. Fluctuación poblacional de ácaros en peral. Temporada 2001-2002. 
difícil encontrar hojas en perales debido al fuerte ataque del chape, insecto que por las condiciones del huerto no se controló químicamente.

A diferencia de lo ocurrido en peral, en manzano las poblaciones fueron más altas en ambas temporadas (Figuras 3 y 4). Durante la temporada 2000-2001 el número de individuos varió de 36 en noviembre hasta 2.066 en enero, mes que además presentó el mayor número de ejemplares, de los cuales 1.367 correspondieron a huevos de ácaros fitófagos. A su vez, los ácaros fitoseidos y acarididos llegaron a 394 individuos. Tetranychus urticae (Koch) alcanzó un máximo de 120 ejemplares, mientras que Panonychus ulmi (Koch) llegó a 56 ejemplares. Cabe destacar que Bryobia rubrioculus (Scheuten) presentó un gran número de ejemplares con un máximo de 120, disminuyendo notablemente en los meses siguientes. Los tideidos en este frutal fueron menos abundantes que el peral, encontrándose un máximo de 206 individuos en marzo (Figura 3). Estos datos coinciden con González (1987) quien indica que a medida que avanzan los meses estivales las poblaciones de ácaros en manzano aumentan al mejorar las condiciones ambientales.

Durante la temporada 2001-2002 en manzano ocurrió un considerable aumento en la mayoría de los ácaros (Figura 4). Así T. urticae llegó a un máximo de 165 ejemplares en el mes de enero. Caso contrario ocurrió con $P$. ulmi que alcanzó un máximo de 35 ejemplares en el mismo mes. Esto se contrapone a Rebolledo et al. (2004) quienes encontraron que el máximo de $P$. ulmi ocurre en marzo.

Los huevos de ácaros fitófagos sufrieron un incremento explosivo llegando hasta los 6.330 en enero y a un mínimo de 146 en abril. B. rubrioculus alcanzó 94 ejemplares en enero y ninguno en mayo. Los acarididos alcanzaron un máximo de 98 individuos en mayo, mes en el cual los otros ácaros casi no presentaron ejemplares adultos.

El efecto de la temperatura media sobre la fluctuación poblacional no presentó diferencias significativas $(\mathrm{P}>0,05)$, por lo cual esta no fue relevante en la variación de las poblaciones de ácaros en los frutales estudiados.

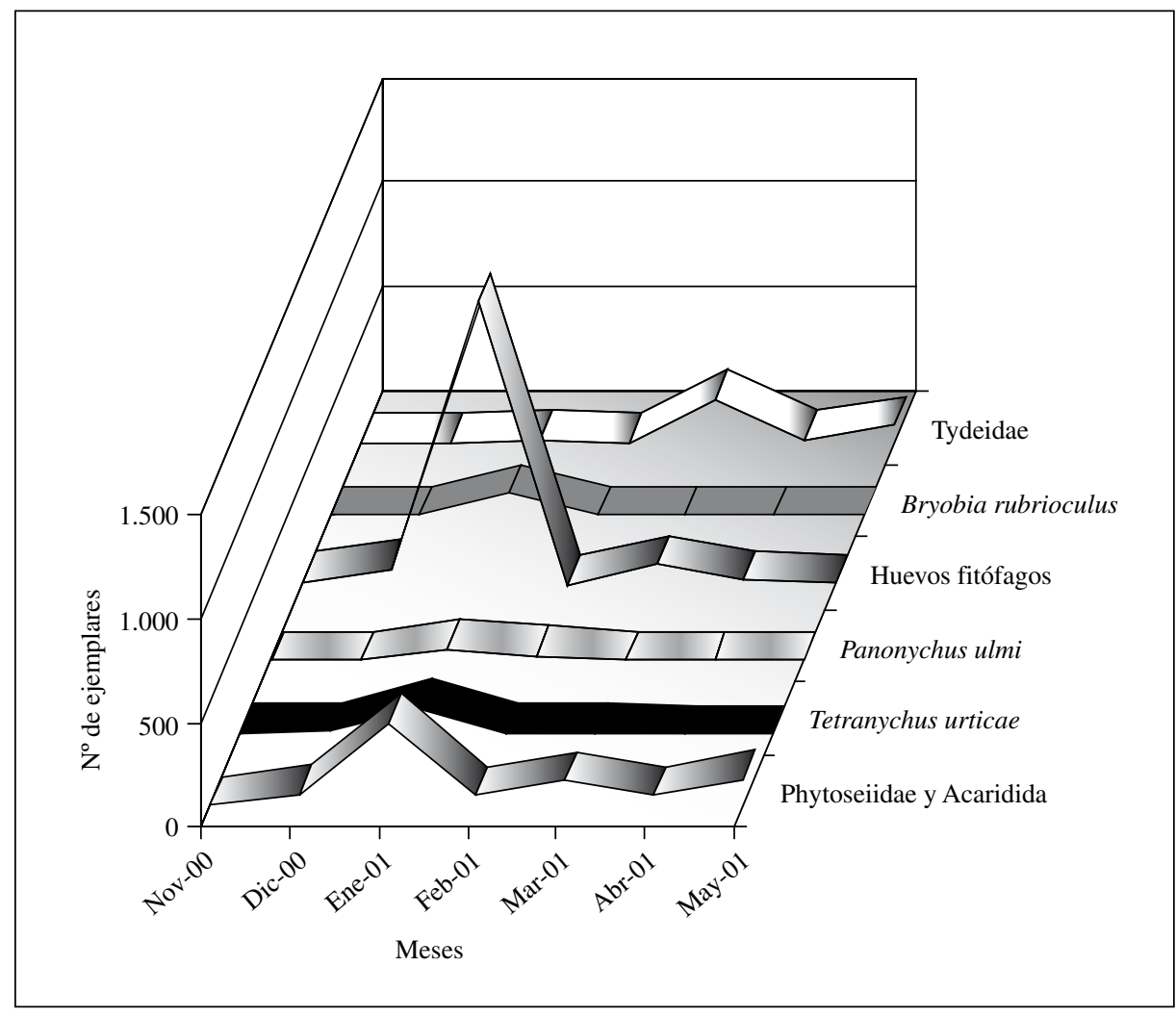

Figura 3. Fluctuación poblacional de ácaros en manzano. Temporada 2000-2001. 


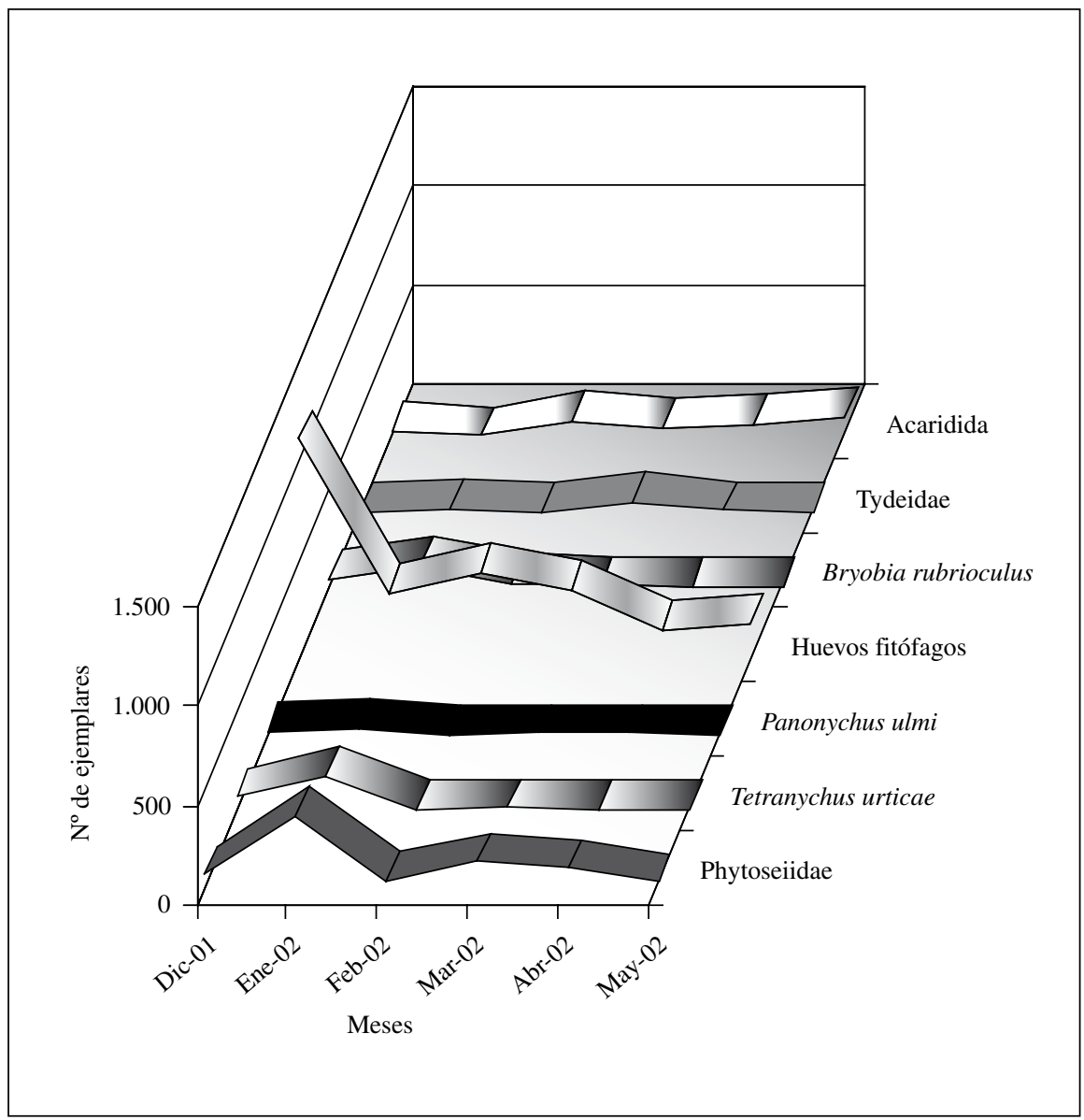

Figura 4. Fluctuación poblacional de ácaros en manzano. Temporada 2001-2002.

Con respecto a la abundancia relativa de ácaros en peral durante la temporada 2000-2001, estuvo marcada por la abundancia de tideidos (Cuadro 1), los cuales según García-Marí et al. (1991) y Ripa y Rodríguez (1999) se alimentan de hongos. Los huevos de ácaros fitófagos, a su vez, superaron en número a los fitoseidos y acarididos, estos últimos presentaron igual porcentaje que T. urticae y P. ulmi, siendo $B$. rubrioculus la especie menos frecuente en este frutal.

En la temporada 2001-2002 los resultados prácticamente no variaron, coincidiendo con la temporada anterior (Cuadro 1). Los tideidos presentaron la mayor abundancia relativa con un $88 \%$; a su vez, fitoseidos, acarididos, T. urticae y $P$. ulmi alcanzaron solamente un $1 \%$; B. rubrioculus representó un 0\% (Cuadro 1).

Los resultados obtenidos pueden estar enmascarados por un fuerte ataque de Caliroa cerasi debido a que prácticamente todas las hojas de los perales estaban dañadas por este insecto, lo cual no dejaría alimento para los ácaros fitófagos y por ende tampoco para los ácaros depredadores.

La abundancia relativa en el caso del manzano fue mayor que en peral (Cuadro 2). Durante la temporada 2000-2001 un 54\% correspondió a huevos de ácaros fitófagos, mientras que acarididos y fitoseidos representaron en su conjunto un $25 \%$. Los tideidos alcanzaron solamente un $10 \%$. A su vez, $T$. urticae y B. rubrioculus alcanzaron un $4 \%$ siendo, de todas maneras, más elevado que en peral. Por su parte, $P$. ulmi alcanzó un $3 \%$, lo cual difiere con Rebolledo et al. (2004) quienes encontraron que esta especie de ácaro fue dominante en manzano coincidiendo con González (1987), quien señaló que $P$. ulmi es el ácaro de mayor abundancia sobre manzano en la zona central del país. 
Cuadro 1

Abundancia relativa de ácaros en peral

\begin{tabular}{|l|l|l|c|}
\hline \multicolumn{1}{|c|}{ Especie } & \multicolumn{1}{|c|}{$\begin{array}{c}\text { Temporada } \\
\mathbf{2 0 0 0 - 2 0 0 1} \\
\text { Porcentaje (\%) }\end{array}$} & \multicolumn{1}{c|}{ Especie } & $\begin{array}{c}\text { Temporada } \\
\mathbf{2 0 0 1 - 2 0 0 2} \\
\text { Porcentaje (\%) }\end{array}$ \\
\hline Phytoseiidae y Acaridida & 1 & Phytoseiidae & 1 \\
Panonychus ulmi & 1 & Acaridida & 1 \\
Tetranychus urticae & 1 & Panonychus ulmi & 1 \\
Bryobia rubrioculus & 0 & Tetranychus urticae & 1 \\
Huevos de fitófagos & 9 & Bryobia rubrioculus & 0 \\
Tydeidae & 88 & Huevos de fitófagos & 9 \\
& & Tydeidae & 88 \\
\hline
\end{tabular}

Cuadro 2

Abundancia relativa de ácaros en manzano

\begin{tabular}{|l|c|l|c|}
\hline \multicolumn{1}{|c|}{ Especie } & \multicolumn{1}{|c|}{$\begin{array}{c}\text { Temporada } \\
\text { 2000-2001 } \\
\text { Porcentaje (\%) }\end{array}$} & Especie & $\begin{array}{c}\text { Temporada } \\
\text { 2001-2002 } \\
\text { Porcentaje (\%) }\end{array}$ \\
\hline Phytoseiidae y Acaridida & 25 & Phytoseiidae & 6 \\
Panonychus ulmi & 3 & Acaridida & 3 \\
Tetranychus urticae & 4 & Panonychus ulmi & 1 \\
Bryobia rubrioculus & 4 & Tetranychus urticae & 3 \\
Huevos de fitófagos & 54 & Bryobia rubrioculus & 2 \\
Tydeidae & 10 & Huevos de fitófagos & 84 \\
& & Tydeidae & 1 \\
\hline
\end{tabular}

En la temporada 2001-2002 se presentó un menor número de ejemplares (Cuadro 2). Solamente los huevos de ácaros fitófagos aumentaron a un $84 \%$; a su vez, T. urticae representó un 3\%, P. ulmi sólo un $1 \%, B$. rubrioculus un $2 \%$ y los fitoseidos un $6 \%$ (Cuadro 2).

Estos resultados difieren de Guajardo (1993), quien en un huerto de manzanos en la X Región de Los Lagos encontró que $P$. ulmi fue la especie predominante en las muestras, no recolectando T. urticae, ni menos B. rubrioculus, a diferencia del presente estudio, el cual precisamente señala que estas arañitas fueron predominantes sobre la arañita roja europea.

En cuanto a la distribución de las arañitas fitófagas, fitoseidos, acarididos, tideidos y huevos de ácaros fitófagos en los diferentes estratos de la planta, se estableció que bajo las condiciones climáticas de Temuco no presentó diferencias significativas en la preferencia por alturas (alta o media) $(\mathrm{P}>0,05)$.
Según la prueba U de Mann-Whitney, se estableció que existen diferencias significativas en la abundancia relativa de estos ácaros entre ambos sustratos vegetales $(\mathrm{P}<0,05)$, lo cual se puede apreciar en las figuras $1,2,3$ y 4 .

\section{CONCLUSIONES}

Las especies de ácaros fitófagos encontradas tanto en peral como en manzano correspondieron a los Tetranychidae Panonychus ulmi (Koch), Tetranychus urticae (Koch), Bryobia rubrioculus (Scheuten) y la única especie de ácaro depredador correspondió a Chileseus camposi González y Schuster (Phytoseiidae). Además se encontraron ácaros pertenecientes a la familia Tydeidae y Acaridae. Se determinaron también diferencias significativas en la abundancia relativa de los ácaros fitófagos y depredadores entre el manzano y peral, 
pero no existieron diferencias significativas en la diversidad de especies encontradas.

La especie Panonychus ulmi, a pesar de ser catalogada por diversos autores como la plaga más importante en manzanos, en el presente estudio se determinó que fue desplazada por Tetranychus urticae y Bryobia rubrioculus, los cuales presentaron una mayor población. Con respecto a la abundancia relativa, esta fue mayor en manzano que en peral, siendo el estado más abundante encontrado el de huevo.

La población de ácaros fitófagos y depredadores presentes en los estratos alto y medio no

\section{LITERATURA CITADA}

APABLAZA, J. 2000. Introducción a la entomología general y agrícola. Ediciones Universidad Católica de Chile. Santiago, Chile. 339 p.

CICHÓN, L.; DIMASI, S.; FERNÁNDEZ, D.; MAGADALEN, J.; RIAL, E. Y ROSSINI, M. 1996. Guía ilustrada para el monitoreo de plagas y enfermedades en frutales de pepita. Departamento de Comunicaciones de la Estación Experimental Agropecuaria Alto Valle de INTA. Río Negro. Argentina. $71 \mathrm{p}$.

COSTA-COMELLES, J.; SANTA MARÍA, A.; FERRAGUT, F. Y GARCÍA-MARÍ, F. 1994. Poblaciones de ácaros en la cubierta vegetal de hueros de manzano. Boletín de Sanidad Vegetal Plagas (España). 20: 339-355.

DORESTE, E. 1984. Acarología. Instituto Interamericano de Cooperación para la Agricultura. Serie Investigación y Desarrollo $N^{o} 11$. Editora Fanny de La Torre. San José, Costa Rica. 391 p.

ESCUDERO. L.; ROSELLÓ, J.; ALEIXANDRE, E.; BRAMARDI, S. Y FERRAGUT, F. 1999. Colonización y dispersión de los ácaros en un ecosistema hortícola protegido: características y factores responsables. Boletín de Sanidad Vegetal Plagas (España). 25: 143-155.

GARCÍA-MARÍ, F.; FERRAGUT, F.; COSTA-COMELLES, J.; LABORDA, R. Y SOTO, T. 1990. Acarología Agrícola. Departamento de Producción Vegetal. Servicio de Publicaciones. Universidad Politécnica de Valencia. Valencia, España. 282 p.

GARCÍA-MARÍ, F.; LLORÉNS, J.; COSTA-COMELLES, J. Y FERRAGUT, F. 1991. Ácaros de las plantas cultivadas y su control biológico. Pisa Ediciones. Valencia, España. $175 \mathrm{p}$.

GARCÍA-MARÍ, F.; COSTA-COMELLES, J. Y FERRAGUT, F. 1994A. Las plagas agrícolas. Ediciones Agropubli, S.L. Valencia, España. 376 p.

GARCÍA-MARÍ, F.; FERRAGUT, F.Y COSTA-COMELLES, J. 1994B. Curso de Acarología Agrícola. Unidad Docente de Entomología Agrícola. Departamento de Producción Vegetal. E.T.S. de Ingenieros Agrónomos. Universidad Politécnica de Valencia, España. 278 p.

GONZÁLEZ, R Y SCHUSTER, R.O. 1962. Especies de la familia Phytoseiidae en Chile. I. (Acarina Mesostigmata). U. de Chile, Facultad de Agronomía. Bol. Téc. 16, 35 p. presentó diferencias significativas para ambos frutales y tampoco se encontró influencia significativa de la temperatura media en la fluctuación poblacional en ambos sustratos vegetales para las dos temporadas.

En términos generales, en ambas temporadas las población de ácaros fitófagos y depredadores, tanto en manzanos como en perales, fue escasa, debido a que el huerto no recibió tratamiento alguno con pesticidas tres años antes del estudio y, por lo menos en perales, influyó la fuerte infestación del chape del cerezo Caliroa cerasi.

GONZÁLEZ, R. 1980. Control integrado de plagas en manzano. Boletín Agrícola Shell (Chile). 40 (2) 1-9.

GONZÁLEZ, R. 1981A. Las arañitas rojas del manzano y del peral. Revista Frutícola (Chile). 2 (1): 3-9.

GONZÁLEZ, R. 1981B. Situación fitosanitaria del manzano y peral, temporada 1980-81. Insectos y ácaros. Revista Frutícola (Chile) 2 (2): 35-43.

GONZÁLEZ, R. 1984. Desarrollo estacional de insectos y ácaros del manzano, 1982-1984. Acaricidas reguladores. Revista Frutícola (Chile) 5 (1): 3-9.

GONZÁLEZ, R. 1987. Acaricidas reguladores del crecimiento contra la arañita roja europea (Panonychus ulmi) en manzanos. Revista Frutícola (Chile). 8 (3): 75-80.

GONZÁLEZ, R. 1989. Insectos y ácaros de importancia agrícola y cuarentenaria en Chile. Editora Ograma S.A. Santiago, Chile. $310 \mathrm{p}$.

GUAJARDO, M. 1993. Ácaros asociados a un huerto de manzano de la X Región y su respuesta frente a insecticidas de distinto sitio de acción. Tesis Ingeniero Agrónomo, Facultad de Ciencias Agrarias. Universidad Austral de Chile (Valdivia, Chile). $135 \mathrm{p}$.

IRAOLA, V.; MORAZA, M. Y BIURRUN, R. 1999. Ácaros tetraníquidos (Acari: Tetranychidae Berlese) y fitoseidos (Acari: Phytoseiidae Berlese) en hojas y cobertura vegetales de perales de Navarra. Boletín de Sanidad Vegetal Plagas (España) 25: 49-58.

KLEIN, C. Y WATERHOUSE, D. 2000. Distribución e importancia de los artrópodos asociados a la agricultura y silvicultura de Chile. Australian Centre for International Agricultural, Research. Monograph $N^{\circ} 68$. Canberra, Australia. $231 \mathrm{p}$.

PÉREZ, I. 1997. Bioecología de los ácaros en la vid. Centro de Publicaciones del Ministerio de Agricultura, Pesca y Alimentación. Madrid, España. Pp. 31-38.

PÉREZ, R., MANSILLA, P. Y XAVIER, A. 1999. Ácaros depredadores fitoseidos en los viñedos de la zona fronteriza de Pontevedra (D.O. Rías Baixas) - Norte de Portugal. Boletín Sanidad Vegetal Plagas (España). 25: 41-48.

PRADO, E. 1991. Artrópodos y sus enemigos naturales asociados a plantas cultivadas en Chile. Publicaciones Estación Experimental La Platina. INIA. Santiago, Chile. 207 p. 
REBOLLEDO, R.; HORMAZÁBAL, O.; AGUILERA, A. Y KLEIN, C. 2004. Infestaciones de la arañita roja europea Panonychus ulmi (Acari: Tetranychidae) en manzanos de la Región de La Araucanía durante la temporada 1996-1997. Rev. Chilena Entomología 30 (1): 65-69.

RIPA, R. Y RODRÍGUEZ, F. 1999. Plagas de cítricos, sus enemigos naturales y manejo. Instituto de Investigaciones Agropecuarias. Ministerio de Agricultura. Centro Experimental de Entomología La Cruz. Santiago, Chile. $151 \mathrm{p}$.

SALAZAR, F.; CARRILLO, R.; AGUILERA. A. Y REBOLLEDO, R. 1998. Antecedentes biológicos de Tetranychus urticae (Koch) (Acari: Tetranychidae) en frambueso cv. Heritage, en Temuco, Chile. Rev. Chilena de Entomología 25: 15-20.
SALAZAR, F.; REBOLLEDO, R.; CARRILLO, R. Y AGUILERA, A. 2000. Factores ambientales y de la planta relacionados con la diapausa de hembras de Tetranychus urticae (Koch) (Acari: Tetranychidae) en Temuco, Chile. Revista Chilena de Entomología. 27: 53-56.

SÁNCHEZ, F. 1994. Control biológico de plagas en invernadero. Agroguías munfi-prensa. Mundi Prensa. Madrid. España. 86 p.

SAZO, L. 1995. Manejo de ácaros fitófagos en frutales de hoja caduca y vides. Sanidad vegetal en frutales y vides. Publicaciones misceláneas agrícolas. Santiago, Chile. 41: 16-19.

VIÑUELA, E. Y JACAS, J. 1998. Acarología en V. De Liñán Entomología Agroforestal. Insectos y ácaros que dañan montes, cultivos y jardines. Ediciones Agrotécnicas F.L. Madrid, España 1309 p. 